\title{
Diagnostic imaging in gynecology
}

\author{
Elzbieta Luczynska' ${ }^{1}$, Zbigniew Kojs ${ }^{2}$ (D) \\ ${ }^{1}$ Electroradiology Department, Jagiellonian University Medical College, Cracow, Poland \\ ${ }^{2}$ M. Sklodowska-Curie Memorial Cancer Center and Institute of Oncology, Cracow Branch, Poland
}

\begin{abstract}
Ultrasound (US), computed tomography (CT) and magnetic resonance imaging (MRI) are the "gold standard" among gynecological imaging methods. They are important diagnostic tools used to determine the site of origin of a pelvic mass and to characterize the adnexal lesions. This paper summarizes the diagnostic performance of ultrasound, computed tomography, and magnetic resonance imaging in various gynecological diseases and tumours diagnostics.
\end{abstract}

Key words: oncology, cancer, computed tomography, magnetic resonance imaging

Ginekologia Polska 2022; 93, 1: 63-69

\section{INTRODUCTION}

Pathological lesions within the female reproductive system organs are classified by clinical, surgical and histopathological criteria. The American College of Radiology (ACR), National Comprehensive Cancer Network (NCCN) and the International Federation of Gynecology and Obstetrics (FIGO) revised the recommendations for diagnostic imaging in cancer detection and staging [1, 2]. European recommendations are also commonly used [3].

Nowadays there are several diagnostic methods used before and after treatment in gynecology, all of them having some advantages and limitations. Accurate diagnosis requires comparison of clinical data and results achieved in diagnostic imaging, and depend on the diagnostic tool and radiologist's level of experience. The gynecological imaging "gold standards" are ultrasound (US), computed tomography (CT) and magnetic resonance imaging (MRI). These methods are useful for determining the site of origin of a pelvic mass and to characterize the adnexal lesions [3-10].

Ultrasonography is the primary imaging technique in patients with clinical symptoms like abnormal uterine bleeding, endocrine disorders, pelvic pain, gynecologic infections and female pelvic lesions. In many cases US has important role in the primary diagnosis of ovarian cancer, the assessment of the tumor in the pelvis and abdominal cavity. For proper diagnosis of pelvic and abdominal organs combination of trans-abdominal (TAUS) and trans-vaginal US (TVUS) is needed. Those techniques may be successfully applied for assessment of an origin of different adnexal masses using IOTA Simple Rules or, in more experienced hands, ADNEX models [11]. It should be remembered that the range of the probe in TVUS is specific and in some cases it does not allow for a full assessment of pelvic pathology, neither for the assessment of pelvic lymph nodes. According to ACR recommendations (2016), if there is a large lesion in pelvis that is outside the ultrasound probe range, transabdominal ultrasound should be performed. For an accurate assessment of both the abdominal cavity and pelvis, we should perform both TAUS and TVUS examinations. TAUS should be performed in two phases, with a full bladder first, to evaluate the uterus and bladder. When the bladder is full, the reproductive organ can be examined, and if we find a tumor, we can possibly assess its relation to adjacent organs, bladder infiltration, ureter infiltration, and find the cause of stagnation in the kidneys (differentiation between infiltration and urolithiasis). Next, the post-voiding examination should be performed to evaluate the parenchymal organs of the abdominal cavity, the aortic space and lymph nodes around the iliac vessels and possible peritoneal tumor cells.

Computed Tomography (CT) is the primary method used to assess the stage of cancer. A CT should be performed before and after intravenous administration of the contrast medium and after oral administration of the contrast medium. Positive oral contrast agents are useful for intestinal and peritoneal tumor cells detection and differentiation of lesions and lymph nodes in the aortic space with intestinal

\footnotetext{
Corresponding author:

Elzbieta Luczynska

Faculty of Medicine, Institute of Nursing and Health Sciences, University of Rzeszow, Poland e-mail: ela.luczynska@op.pl
} 
loops. Examination after oral administration of contrast medium is particularly useful in debilitated patients. Negative oral contrast agents are useful for calcified tumor detection. Lesions in the peritoneum with more than $10 \mathrm{~mm}$ of width in the short axis are visible on CT scan. Peritoneal tumor implants smaller than $10 \mathrm{~mm}$ may be difficult to detect, especially if they are not accompanied by ascites. If lymph nodes short axis is more than $10 \mathrm{~mm}$, or in case of morphological changes - such as rounded node shape or necrosis in the node, lymph node metastases are also suspected. The disadvantages of CT are the patient's exposure to ionic radiation, possible allergic reaction to iodine contrast agents and worse soft tissue visualisation in comparison to MRI.

Magnetic Resonance Imaging (MRI) is a diagnostic modality that provides much better images of soft tissue than CT scans. Due to the possibility of tissue differentiation, MRI allows for better estimation of the primary tumor extent and adjacent structures infiltration assessment. Local infiltration assessment on MRI should be performed in planes - transverse, coronal and sagittal to the long axis of the uterus. Dynamic Contrast Enhancement (DCE) MRI imaging after intravenous contrast injection is useful in detecting well vascularized tumors. Chemical shift imaging or sequences with fat suppression are excellent for detecting fat within lesions. Post-contrast T1 weighted images with fat saturation enable peritoneal lesions detection. Diffusion Weighted Imaging (DWI) is sensitive to the movement of water molecules at the cellular level. Malignant tumors, for example, are more likely to show a high intensity signal on DWl at a high b (1000) value and are visible as dark on ADC (Apparent Diffusion Coefficient) maps. MRI with dynamic contrast enhancement combined with DWI allows for better characterization and assessment of tumor stage and extent including detection of subtle peritoneal disease, as well as assessment of tumor response and recurrence. DWI and the corresponding ADC maps improve tumor characteristics and stage estimation in patients with endometrial and cervical cancer, but are less specific in distinguishing between benign and malignant myometrial and ovarian tumors. In case of kidney dysfunction, when using gadolinium based contrast agent is not possible, DWI constitutes a very important part of MRI examination.

Despite the advantages (no ionic radiation, better soft tissue visualization) MRI has some disadvantages — such as low availability, higher cost, long image acquisition time leading to motion artefacts, reduced patient cooperation and MRI safety problems [12].

\section{OVARIAN CANCER, FALLOPIAN TUBES, PRIMARY PERITONEAL CANCERS}

Ultrasound (US) is the first choice examination performed in patients with suspected ovarian cancer. Specific features of ovarian tumors visible on US are: enlargement of ovarian dimensions above $5 \mathrm{~cm}$, presence of internal partitions in the tumor, papillary growths on the inner surface of the tumor, complex structure (solid-cystic), free fluid in Pouch of Douglas ( $\max 5 \mathrm{~mm}$ ), poor tumor mobility, infiltration of adjacent organs. In some cases, TAUS allows for visualization of solid hypoechoic peritoneal lesions and infiltrations to the adjacent tissue.

In case of ovarian cancer, NCCN set the following recommendations: CT with iodine contrast agent of the chest, abdomen and pelvis as well as abdominal and pelvic MRI for primary diagnosis and follow-up imaging. According to the ACRAC the most important examination is $C T$ with contrast enhancement of the abdomen and pelvis for both pre-treatment assessment and follow-up (Fig. 1). MRI has great value in assessing the staging of ovarian cancer. According to the recommendations of ESUR (European Society of Urogenital Radiology) MRI is recommended in case of ambiguous ultrasound results. The IOTA (International Ovarian Tumor Analysis) guideline suggests the malignant character of the lesion visualized in MRI if its size is more than $4 \mathrm{~cm}$, its morphology is complex (solid and cystic) with solid enhancing component, contains thick septations $>3 \mathrm{~mm}$, has papillary projections or central necrosis. Malignant lesions usually have Type 3 dynamic contrast curve and possible additional findings, like ascites, lymph node enlargement, peritoneal carcinomatosis or organ invasion.

The characteristic symptoms strongly suggestive of malignancy on CT scan in the first stage of ovarian cancer include: wall irregularities or thickening $>3 \mathrm{~mm}$, enhancing solid components, abdominal wall tumors and papillae. Serous cystic adenocarcinomas may contain microcalcifications. Serous cystic adenomas are usually small and single-celled, whereas mucous cystic adenomas are larger multicellular clusters [13]. Mucinous tumors are attenuated on CT and have heterogeneous signal intensity on

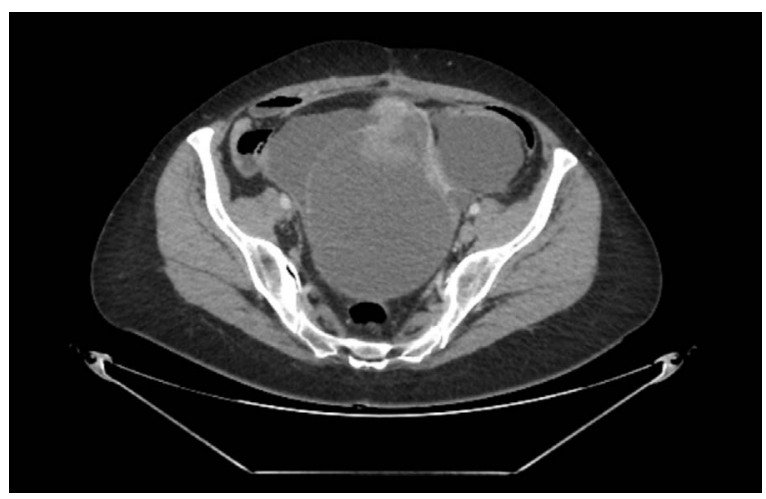

Figure 1. CT image of ovarian cancer. The tumor is seen to consist of both solid and cystic components. The solid component enhanced after intravenous administration of contrast medium 
T1-weighted and T2-weighted MRI images, which results in the appearance of "stained glass". Primary malignant tumors of the fallopian tubes may have the appearance of solid, mixed solid / cystic masses or papillary projections in dilated fallopian tubes that enhance less than myometrium on CT and MRI. The adnexa of uterus masses with the appearance of "sausages" associated with hydrosalpinx and the presence of intrauterine fluid strongly indicate malignant fallopian tube cancer.

MRI is a very good diagnostic method for assessing whether the ovarian cancer is suitable for surgery. Its sensitivity is $92 \%$, specificity $85 \%$ [14]. It appears to be a much better method than CT in evaluating the local stage of ovarian cancer, with sensitivity of $55 \%$, specificity $88 \%$ and accuracy $89 \%$. At higher stages of ovarian cancer, peritoneal metastasis is manifested in the form of nodules or "plaques" in the peritoneum, fluid in the peritoneal cavity, mesenteric fat infiltration and mesenteric lymph or node enlargement in para-aortic area.

In grade II and III of the disease, DCE-MRI with DWI improves the detection of pelvic and peritoneal tumor cells. Local tumor involvement can be suspected if there is a lack of adipose tissue between the tumor and adjacent pelvic structures, irregular contact surface between the tumor and the adjacent structure, less than $3 \mathrm{~mm}$ distance between the tumor and the pelvic wall and ureteral obstruction and dislocation/closure of the iliac vessels.

MRI outperforms CT in cancer infiltration assessment. Symptoms of bowel infiltration are wall thickening and its irregular shape. Metastatic retroperitoneal, pelvic and inguinal lymph nodes are also possible. Peritoneal tumor manifests as peritoneal wall enhancement and thickening, peritoneal tuberosity/mesentery, omental caking, and/or mesenteric infiltration.

Presence of ascites should be treated as suggestive of peritoneal disease. Oral administration of the contrast agent may improve detection. CT has limitations in the visualization of peritoneal tumor implants smaller than $1 \mathrm{~cm}$, especially if ascites is not present. The correct path of peritoneal fluid circulation involves the pelvis and the diaphragm. The role of MDCT is limited in detecting small peritoneal metastases. If the peritoneal metastasis is greater than $1 \mathrm{~cm}$, the MDCT sensitivity is 85 to $93 \%$ and the specificity is $91-96 \%$. For metastases with a diameter less than $1 \mathrm{~cm}$, the MDCT sensitivity decreases to $25-50 \%$.

In stage IV of the disease, cancer is likely to spread in certain ways including transperitoneal, lymphatic or hematogenous way. The most common sites of ovarian cancer distant metastases include lungs and liver. Cytological evaluation is necessary to confirm malignant pulmonary effusion, however, the presence of thickening, tuberosity or masses suggests malignancy.
Another imaging modality useful in ovarian cancer detection is PET (Positron-Emission Tomography). This examination is performed if recurrence is suspected, with growing marker values observed with no corresponding pathological process visible on diagnostic images. PET/CT is particularly useful in distinguishing between patients in stage IIIC-IV and I-IIIb and in this case the specificity is $91 \%$, sensitivity $100 \%$ and accuracy $98 \%$ compared to $64 \%, 97 \%$ and $88 \%$ of CT alone. PET/CT has high sensitivity in detecting peritoneal metastases larger than $1 \mathrm{~cm}$ and lymph nodes larger than $7 \mathrm{~mm}[14]$.

\section{ENDOMETRIAL CANCER}

TVUS is mainly applied for evaluation of endometrium among women with symptoms of bleeding in post-menopausal period. The screening diagnosis obtained on TVUS is based on the measurement of endometrium thickness. There is a notable thickening of endometrium visible on US examination in case of endometrial cancers [15]. Main limitations of TVUS are: operator dependence, limited vision field, danger of overestimation of infiltration extent and lymphatic vessels area, low efficiency of the method in predicting cancer extent, evaluating cervix infiltration and lymph nodes assessment. However, there are literature data confirming high accuracy of US. Recently performed study conducted in Sweden involved a large group of patients ( 850 women with endometrioid endometrial cancer). The examinations performed by ultrasound experts revealed high accuracy of myometrial invasion ultrasound assessment.

$A C R$ and NCCN definitely recommend MRI examination of pelvis with and without intravenously administered contrast agent to assess the depth of myometrial invasion and tumor extent in order to establish treatment plan for individual patients (Fig. 2) [16]. Overall accuracy of MRI in cancer staging is $83-92 \%$, while sensitivity, specificity and accuracy of MRI in evaluation of cervical infiltration on T2-weighted images are $100 \%, 87 \%$ and $90 \%$ and on T1-weighted images after contrast administration - 100\%, 95\% and 96\% [17].

The depth of myometrial invasion in endometrial cancer is an important morphological prognostic factor correlating with tumor grade, presence of lymph node metastases, and overall patient survival. MRI with contrast medium administration is superior to CT with contrast enhancement, US and MRI without contrast administration in assessment of local stage of disease. It is possible to perform pelvic CT with contrast instead of MRI in case of lack of MRI equipment availability. ACR does not recommend tomography without contrast administration. Sensitivity and specificity of CT in myometrium infiltration assessment ranges from 40 to $83 \%$ and respectively 42 to $75 \%$, while accuracy in myometrium and cervix infiltration assessment is 81 to $95 \%[1,18]$. 


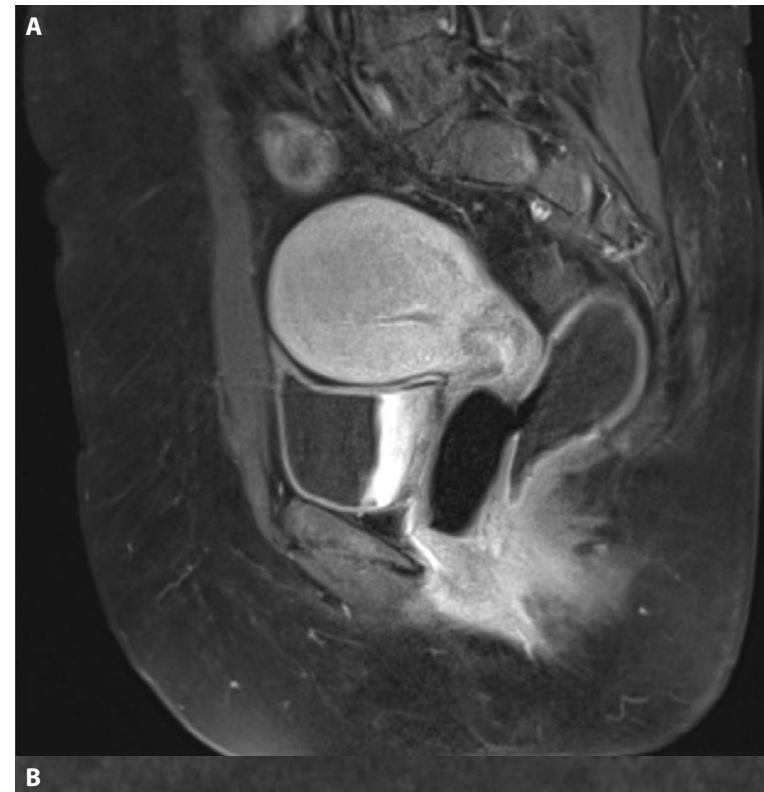

B

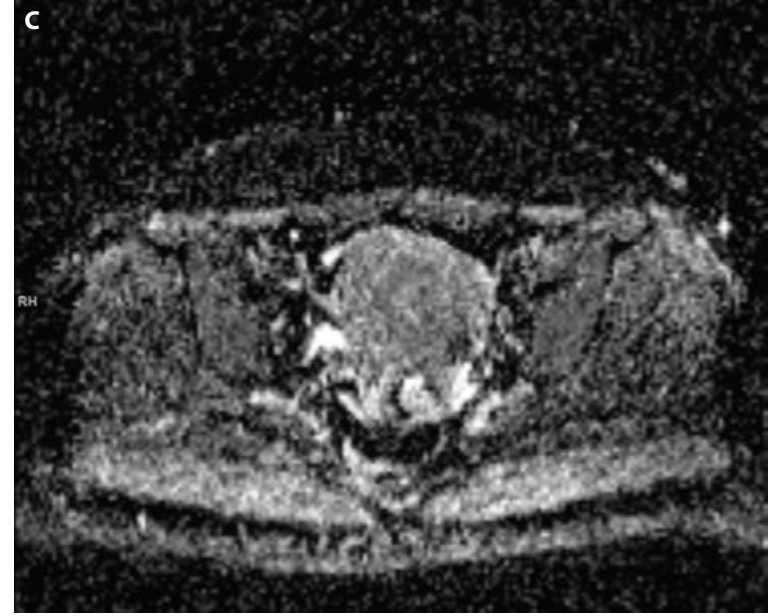

Figure 2. MR images of uterine cancer; $\mathbf{A}$. Example MR image in transversal plane, enlarged uterus, thickened endometrium; B. Sagittal MR image of the pelvis, thickened endometrium is visible; there is no border between endometrium and myometrium. Tumor infiltrates more than 50\% myometrium; C. DWI and corresponding $\mathrm{ADC}$ map, the area of restricted diffusion is visible
Both CT with contrast enhancement and abdomen and pelvis MRI are useful in lymph nodes evaluation. For highly malignant tumors it is recommended to perform chest CT for possible metastases assessment.

In case of tumors classified as stage I to IIIb MRI of the pelvis is superior to CT or PET in evaluation of local extent of disease. According to the American Joint Committee on Cancer (AJCC) and FIGO staging system, stage I tumors are divided into: stage I - cancer is found only in the uterus, and it has not spread to other parts of the body; stage IA — cancer is found only in the endometrium or less than one-half of the myometrium; stage IB - spread to one-half or more of the myometrium. Stage II tumors involve the cervical stroma causing widening of the internal os. Stage IIIA and B tumors comprise local invasion of the serosa, adnexa, vagina, and parametrium, while stage IIIC1 involves cancer spread to the regional pelvic lymph nodes and stage IIIC2 - spread to the para-aortic lymph nodes with or without spread to the regional pelvic lymph nodes. Stage IV, also divided into $A$ and $B$ means that cancer has metastasized to the rectum, bladder, and/or distant organs beyond the true pelvis. The accuracy of determining myometrial and cervical invasion increases with the addition of dynamic contrast imaging and is the most advantageous when performed 2 to 4 minutes after contrast medium administration. In case of contraindications for contrast administration, DWI is applied with similar sensitivity and specificity to enhanced imaging in myometrium invasion detection.

At present, for assessment of lymph nodes invasion and distant extent of the disease MRI and abdominal CT with contrast enhancement is recommended for initial management planning. Invasion of pelvic, para-aortic, or inguinal lymph nodes has an impact on prognosis. Accuracy of DWI in lymph nodes evaluation is similar to MRI with contrast enhancement.

Endometrial cancer is characterized by intensive FDG uptake, while PET/CT are not significant enough to be taken into consideration in initial staging of early endometrial cancer due to limited spatial resolution and physiological uptake in pre-menopausal women. Sensitivity, specificity and accuracy of PET/CT in lymph nodes metastases detection regardless of lymph node size is 53\%, $99 \%$ and $98 \%$, respectively [19] maximum standardized uptake value (SUVmax).

\section{UTERINE SARCOMA}

In case of uterine sarcoma CT or MRI of the abdomen and pelvis is recommended, although MR imaging is preferred for local extension evaluation or in patients with incidentally detected tumors with incomplete resections after myomectomy or morcellation. Chest CT is also recommended due to frequent cases of lung metastases. Recommendations 
after therapy include chest, abdomen and pelvis CT. Pelvis $M R I$ is also suggested if recurrence of the disease is suspected. Due to the fact that MRI enables visualisation of tissue differentiation and is performed in three planes, it allows for distinguishing between recurrence and normal pelvic structures.

MRI examination also facilitates differentiating between sarcoma subtypes. It enables determining tumor location and contour, as well as presence of hemorrhagic, necrotic and cystic components. It is difficult to differentiate leiomyosarcomas from leiomyomas with imaging examination, if rapid growth or visible metastases are not present. Leiomyosarcoma is typically visible as infiltrating myometrial mass with irregular or ill-defined margins. On imaging after contrast administration, peripheral enhancement with central heterogeneous signal intensity from necrosis and haemorrhage is present.

Endometrial stromal sarcoma (ESS) and undifferentiated sarcoma (UES) most often appear as polypoid endometrial masses with wormlike bands of low T2-weighted signal intensity corresponding to regions of preserved myometrium. Sometimes they have more marginal nodularity resulting from their tendency to infiltrate lymph vessels. Adenosarcomas are mixed tumors, including benign and malignant components, frequently appearing as complex, multiseptated, cystic, and solid polypoid endometrial masses.

The usefulness of DWI is limited due to significant overlap of ADC values between benign leiomyomas and sarcomas. However, if patients are unable to receive contrast, this examination should be applied to support diagnosis.

\section{CERVICAL CANCER}

In 2018 an updated FIGO staging system for uterine cervical cancer was introduced [20]. Imaging examinations became an important part of staging process, being a reliable source of information pertaining to cancer spread complementary to this obtained in clinical examination. Imaging examinations are indicated in patients with FIGO stage IB disease or greater. Due to important prognostic factors obtained in imaging, such as tumor size, parametrial spread, and lymph node metastases it is considered as highly valuable. Contrast enhanced pelvic MRI is performed in patients who need preoperative planning. For FIGO stage II disease or higher, chest, abdomen, and pelvis $\mathrm{CT}$ is recommended in order to detect possible metastases and contrast-enhanced pelvic MRI to assess local extent of disease (Fig. 3) [21].

To evaluate the primary tumor on MRI imaging, both T2-weighted large field-of-view sequences and the small field-of-view sections obtained perpendicular to the endocervical canal are recommended. To enable better evaluation of the vaginal fornices for local spread, application

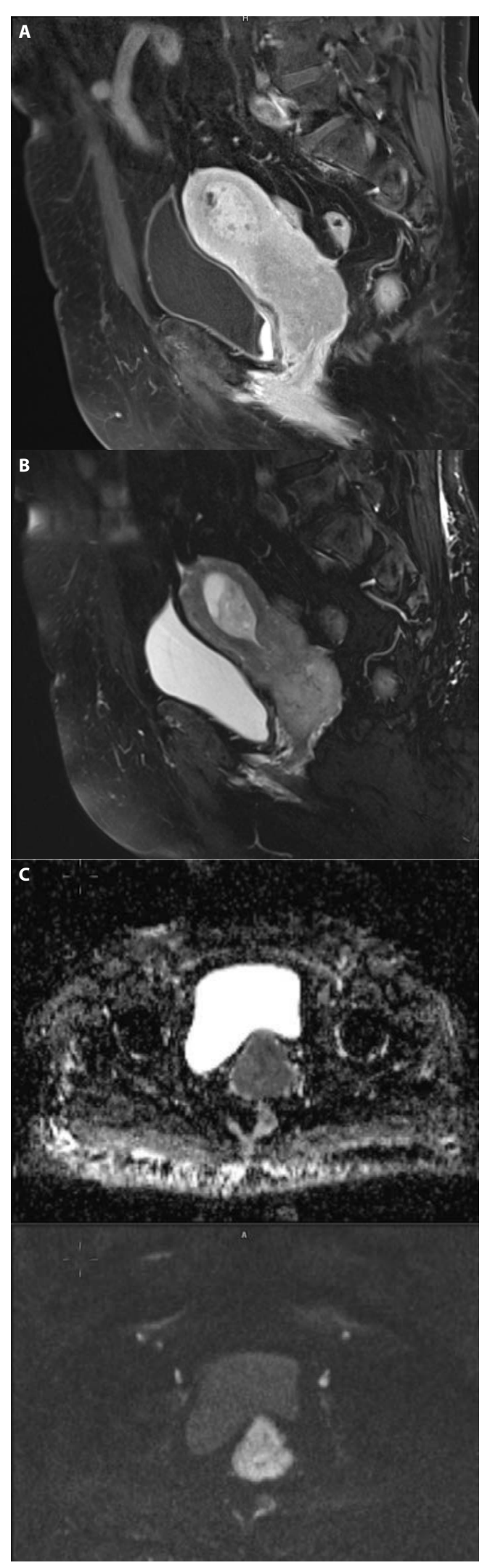

Figure 3. Example MR images of cervical cancer; A. T1-weighted image in sagittal plane after intravenous contrast injection, visible infiltration of uterus and vagina; B. T2 sagittal image of cervical cancer; $\mathbf{C}$. DWI and corresponding ADC map - the area of restricted diffusion is visible 
of vaginal gel is recommended. Contrast administration improves detection of small tumors, depth of stromal invasion, and bladder or rectal wall invasion. As cervical cancer normally exhibits lower ADC values than the normal cervix, DWI can also be helpful in tumor detection.

Stage I disease means that carcinoma is strictly confined to the cervix. It is further subdivided into: stage IA which represents invasive carcinoma that can be diagnosed only with microscopy, with maximum depth of invasion $<5 \mathrm{~mm}$ (IA1 - stromal invasion $<3 \mathrm{~mm}$ and IA2 - stromal invasion $\geq 3 \mathrm{~mm}$ and $<5 \mathrm{~mm}$ in depth) and IB - invasive carcinoma confined to the uterine cervix, with measured deepest invasion $\geq 5 \mathrm{~mm}$ (also divided in new staging system into: IB1 - tumor measures $<2 \mathrm{~cm}$, IB2 $\geq 2 \mathrm{~cm}$ and $<4 \mathrm{~cm}$ and IB3 $\geq 4 \mathrm{~cm}$ in greatest dimension).

On MR imaging, the normal cervix has a trilaminar appearance on T2-weighted imaging with endocervical mucosa of a high signal intensity, a low signal intensity fibromuscular stroma, and an intermediate signal intensity outer smooth muscle layer. On T2-weighted sequences tumors appear intermediate to high signal intensity relative to the hypointense middle stromal layer and have variable enhancement pattern on sequences received after contrast administration.

Stage II disease extends beyond the uterus but does not involve the pelvic sidewalls or the lower one-third of the vagina.

Stage IIA disease extends into the upper two-thirds of the vagina or to the pelvic wall. Tumors are subdivided into stage IIA 1 and IIA 2 regarding their size greater or smaller than $4 \mathrm{~cm}$. IIB disease means parametrial invasion, but not up to the pelvic wall. MRI findings highly indicative of parametrial invasion include disruption of the low T2-weighted signal intensity inner cervical stromal ring and/or presence of nodular or irregular tumor extending into the parametrium. It is possible to exclude parametrial invasion if the inner cervical stromal rim is thicker than $3 \mathrm{~mm}$. Such a finding is called a hypointense rim sign. In case of parametrial invasion surgical procedure is not taken into consideration.

Accuracy of MRI in cervical cancer staging (operable or inoperable lesion) equals $75-96 \%$. MRI is highly accurate in evaluating tumor size in correlation with histopathological examination, 70-90\%. MRI has high NPV in parametrial invasion assessment, 94-100\% [22]. DWI with ADC sequences may be helpful to distinguish benign reactive changes or stromal edema from true tumor invasion. On CT findings suggestive of parametrial invasion include encasement of the ureters, presence of vasculature, and thickening of the uterosacral ligaments.

Findings present in stage III involves the lower third of the vagina and/or extends to the pelvic wall and/or causes hydronephrosis or non-functioning kidney and/or involves pelvic and/or para-aortic lymph nodes. In stage IIIA, the tumor extends to the lower one-third of the vagina. Stage IIIB disease involves extension to the pelvic sidewall or to the ureters. When the tumor is within $3 \mathrm{~mm}$ of the pelvic sidewall musculature, it can be suggestive of pelvic sidewall involvement. In 2018 system another category appeared: IIIC — meaning involvement of pelvic and/or para-aortic lymph nodes, irrespective of tumor size and extent (IIIC1 — pelvic lymph node metastasis only and IIIC2 - para-aortic lymph node metastasis).

Stage IV disease includes invasion of the urinary bladder, rectum, or tumor extension beyond the true pelvis. Stage IVA means invasion of the bladder or rectum and is suggested by following findings: loss of the perivesical or perirectal fat plane, disruption of the normal muscle low T2-weighted signal intensity, asymmetric nodular wall thickening, intraluminal mass, or fistula. If tumor extends beyond the pelvis and spreads to distant organs, is classified as stage IVB Lymph node metastasis is a significant prognostic factor in cervical cancer staging and is associated with a reduction in survival. It has been added to FIGO classification (2018).

At the beginning, cervical lymph nodes drain to the parametrial nodes and then spread to the external iliac, internal iliac, presacral, common iliac, and para-aortic lymph nodes. Specificity of CT and MRI in the detection of lymph node metastasis is greater than $90 \%$ but sensitivity is less than $60 \%$.

Surgical excision of the lymph nodes, either elective or selective still remains the standard procedure in diagnosing lymph nodes metastasis. Short-axis diameter larger than $1 \mathrm{~cm}$ is the main criterion used for identification of abnormal nodes. It is impossible to reliably detect micrometastases with CT or MR imaging [23]. Additional characteristics such as a rounded shape, irregular margins, clusters of multiple small lymph nodes, signal intensity similar to the primary tumor, and necrosis are suggestive of metastases. What is more, lymph nodes positive for metastasis show increased tracer uptake on PET/CT examination being localised on the drainage pathway from uterine cervical cancer.

$\mathrm{PET} / \mathrm{CT}$ and PET/MRI are highly accurate in lymph nodes metastases diagnostics, 85-95\% [24]. Another advantage is sensitivity of PET/CT in lymphadenopathy detection, with PET having higher sensitivity than MRI (75\% to $56 \%$ ) and CT (58\%). It was proven, that while CT and MRI demonstrate similar diagnostic performance, PET/CT outperforms both in lymphadenopathy detection.

$\mathrm{PET} / \mathrm{MRI}$ is a chosen method for primary tumor evaluation with sensitivity of $99 \%$ [25]. This examination, according to the recent literature data and updated FIGO system is a vital method in local and general cervical cancer staging.

\section{SUMMARY}

Ultrasounds, especially TVUS as a widespread and fundamental skill among gynecology specialists is considered 
as the initial examination in pelvis viscera diagnostics. It may also have a substantial role in staging while performed by a US expert in dedicated cancer units. For local staging of a uterine body and a cervix cancer MR imaging is the best choice, while for assessment of retroperitoneal space of above mentioned malignancies and for an ovarian cancer staging the method of choice is CT. Additionally, combined imaging modalities, such as PET/CT or PET/MRI are promising tools improving staging accuracy.

\section{Conflict of interest}

All authors declare no conflict of interest.

\section{REFERENCES}

1. Kang SK, Reinhold C, Atri M, et al. Expert Panel on Women's Imaging:, Expert Panel on Women's Imaging:. ACR Appropriateness Criteria Ovarian Cancer Screening. J Am Coll Radiol. 2017; 14(11S): S490-S499, doi: 10.1016/j.jacr.2017.08.049, indexed in Pubmed: 29101987.

2. Gomes Ferreira M, Sancho de Salas M, González Sarmiento R, et al. Changes in the Management and Prognosis of Ovarian Cancer Due to the New FIGO and WHO Classifications: A Case Series Observational Descriptive Study. Seven Years of Follow-up. Int J Gynecol Cancer. 2018; 28(8): 1461-1470, doi: 10.1097/IGC.0000000000001331, indexed in Pubmed: 30045138.

3. Javadi S, Ganeshan DM, Qayyum A, et al. Ovarian Cancer, the Revised FIGO Staging System, and the Role of Imaging. AJR Am J Roentgenol. 2016; 206(6): 1351-1360, doi: 10.2214/AJR.15.15199, indexed in Pubmed: 27042752

4. Lenhard MS, Burges A, Johnson TRC, et al. PET-CT in recurrent ovarian cancer: impact on treatment planning. Anticancer Res. 2008; 28(4C): 2303-2308, indexed in Pubmed: 18751410.

5. Grisaru D, Almog B, Levine $C$, et al. The diagnostic accuracy of $18 \mathrm{~F}$-fluorodeoxyglucose $\mathrm{PET} / \mathrm{CT}$ in patients with gynecological malignancies. Gynecol Oncol. 2004; 94(3): 680-684, doi: 10.1016/j.ygyno.2004.05.053, indexed in Pubmed: 15350358.

6. Son $\mathrm{H}$, Khan SM, Rahaman J, et al. Role of FDG PET/CT in staging of recurrent ovarian cancer. Radiographics. 2011; 31(2): 569-583, doi: 10.1148/rg.312105713, indexed in Pubmed: 21415197.

7. Théodore $\mathrm{C}$, Levaillant JM, Capmas $\mathrm{P}$, et al. MRI and Ultrasound Fusion Imaging for Cervical Cancer. Anticancer Res. 2017; 37(9): 5079-5085, doi: 10.21873/anticanres.11925, indexed in Pubmed: 28870937.

8. Devine C, Viswanathan C, Faria S, et al. [18F]-2-Fluoro-2-Deoxy-D-glucose-PET Assessment of Cervical Cancer. PET Clin. 2018; 13(2): 165-177, doi: 10.1016/j.cpet.2017.11.004, indexed in Pubmed: 29482748.

9. Brito Pires NM, Godoi ET, Oliveira DC, et al. Impact of pelvic magnetic resonance imaging findings in the indication of uterine artery embolization in the treatment of myoma. Ginekol Pol. 2017; 88(3): 129-133, doi: 10.5603/GP.a2017.0024, indexed in Pubmed: 28397200.

10. Berek JS, Matsuo K, Grubbs BH, et al. Multidisciplinary perspectives on newly revised $2018 \mathrm{FIGO}$ staging of cancer of the cervix uteri. J Gynecol Oncol. 2019; 30(2): e40, doi: 10.3802/jgo.2019.30.e40, indexed in Pubmed: 30740962.

11. Van Calster B, Van Hoorde K, Valentin L, et al. International Ovarian Tumour Analysis Group. Evaluating the risk of ovarian cancer before surgery using the ADNEX model to differentiate between benign, borderline, early and advanced stage invasive, and secondary metastatic tumours: prospective multicentre diagnostic study. BMJ. 2014; 349: g5920, doi: 10.1136/bmj.g5920, indexed in Pubmed: 25320247.

12. Sala $E$, Wakely $S$, Senior $E$, et al. MRI of malignant neoplasms of the uterine corpus and cervix. AJR Am J Roentgenol. 2007; 188(6): 1577-1587, doi: 10.2214/AJR.06.1196, indexed in Pubmed: 17515380.

13. Cerci ZC, Sakarya DK, Yetimalar MH, et al. Computed tomography as a predictor of the extent of the disease and surgical outcomes in ovarian cancer. Ginekol Pol. 2016; 87(5): 326-332, doi: 10.5603/GP.2016.0002, indexed in Pubmed: 27304646.

14. Suppiah S, Chang WL, Hassan HA, et al. Systematic Review on the Accuracy of Positron Emission Tomography/Computed Tomography and Positron Emission Tomography/Magnetic Resonance Imaging in the Management of Ovarian Cancer: Is Functional Information Really Needed? World J Nucl Med. 2017; 16(3): 176-185, doi: 10.4103/wjnm. WJNM_31_17, indexed in Pubmed: 28670174.

15. Wahl AO, Gaffney DK, Jhingran A, et al. ACR Appropriateness Criteria ${ }^{\star}$ Adjuvant Management of Early-Stage Endometrial Cancer. Oncology (Williston Park). 2016; 30(9): 816-822, indexed in Pubmed: 27633412.

16. Hricak $\mathrm{H}$, Chen $\mathrm{M}$, Coakley FV, et al. Complex adnexal masses: detection and characterization with MR imaging--multivariate analysis. Radiology. 2000; 214(1): 39-46, doi: 10.1148/radiology.214.1.r00ja3939, indexed in Pubmed: 10644099 .

17. Rieber A, Nüssle K, Stöhr l, et al. Preoperative diagnosis of ovarian tumors with MR imaging: comparison with transvaginal sonography, positron emission tomography, and histologic findings. AJR Am J Roentgenol. 2001; 177(1): 123-129, doi: 10.2214/ajr.177.1.1770123, indexed in Pubmed: 11418411.

18. Pandharipande PV, Lowry KP, Reinhold C, et al. Expert Panel on Women's Imaging:. ACR Appropriateness Criteria Ovarian Cancer Screening. J Am Coll Radiol. 2017; 14(11S): S490-S499, doi: 10.1016/j.jacr.2017.08.049, indexed in Pubmed: 29101987.

19. Yanarateş $A$, Budak E. Prognostic role of PET/CT in endometrial cancer. Ginekol Pol. 2019; 90(9): 491-495, doi: 10.5603/GP.2019.0085, indexed in Pubmed: 31588544

20. Lee SI, Atri M. 2018 FIGO Staging System for Uterine Cervical Cancer: Enter Cross-sectional Imaging. Radiology. 2019; 292(1): 15-24, doi: 10.1148/radiol.2019190088, indexed in Pubmed: 31135294.

21. Siegel CL, Andreotti RF, Cardenes HR, et al. American College of Radiology. ACR Appropriateness Criteria ${ }^{\circledR}$ pretreatment planning of invasive cancer of the cervix. J Am Coll Radiol. 2012; 9(6): 395-402, doi: 10.1016/j. jacr.2012.02.021, indexed in Pubmed: 22632665.

22. Epstein E, Testa A, Gaurilcikas A, et al. Early-stage cervical cancer: tumor delineation by magnetic resonance imaging and ultrasound - a European multicenter trial. Gynecol Oncol. 2013; 128(3): 449-453, doi: 10.1016/j.ygyno.2012.09.025, indexed in Pubmed: 23022593.

23. Choi HJ, Ju W, Myung SK, et al. Diagnostic performance of computer tomography, magnetic resonance imaging, and positron emission tomography or positron emission tomography/computer tomography for detection of metastatic lymph nodes in patients with cervical cancer: meta-analysis. Cancer Sci. 2010; 101(6): 1471-1479, doi: 10.1111/j.134 9-7006.2010.01532.x, indexed in Pubmed: 20298252.

24. Kidd EA, Siegel BA, Dehdashti F, et al. Lymph node staging by positron emission tomography in cervical cancer: relationship to prognosis. J Clin Oncol. 2010;28(12):2108-2113, doi: 10.1200/JCO.2009.25.4151, indexed in Pubmed: 20308664.

25. Trial M, Atri M, Epid D, et al. Identification of Distant Metastatic Disease in Uterine Cervical and Endometrial Cancers with FDG PET / CT. Radiology 000. 2018. 